\title{
Entanglement between total intensity and polarization for pairs of coherent states
}

\author{
Carlos Sanchidrián-Vaca and Alfredo Luis* \\ Departamento de Óptica, Facultad de Ciencias Físicas, Universidad Complutense, 28040 Madrid, Spain
}

(Received 21 January 2018; published 9 April 2018)

\begin{abstract}
We examine entanglement between number and polarization, or number and relative phase, in pair coherent states and two-mode squeezed vacuum via linear entropy and covariance criteria. We consider the embedding of the two-mode Hilbert space in a larger space to get a well-defined factorization of the number-phase variables. This can be regarded as a kind of protoentanglement that can be extracted and converted into real particle entanglement via feasible experimental procedures. In particular this reveals interesting entanglement properties of pairs of coherent states.
\end{abstract}

DOI: 10.1103/PhysRevA.97.043810

\section{INTRODUCTION}

Entanglement is a distinguishing feature disclosing rather fundamental nonclassical phenomena, as well as a powerful resource for emerging quantum technologies. Entanglement has many facets so it may be convenient to distinguish different types. In this work we distinguish between particlelike versus variablelike entanglement, also referred to as intersystem versus intrasystem, respectively, as introduced in Ref. [1]. This might be related as well with the distinction between intermode and intramode correlations in quantum metrology [2]. By particlelike we mean entanglement between variables belonging to two physically different systems, that is, local observables. By variablelike we mean entanglement between commuting variables of a single system, without any clear subsystem splitting.

In this work we focus on variablelike entanglement for pairs of coherent states. We show that there is entanglement between total number and relative-phase or polarization variables, revealed by linear entropy and covariance criteria for pure states. Pure states are maximal preparations, so all fluctuations and correlations might be ascribed to a quantum origin. The key point is that entanglement is equivalent to nonclassicality in the traditional sense of lack of bona fide phase-space joint distribution [3]. Thus entanglement in pairs of Glauber coherent states is consistent with previous evidences of nonclassical behavior as revealed by negativity of number-phase Wigner functions [4], nonclassical statistics in photon number detection [5], and unbalanced double homodyne detection [6], as well as other results [7]. This also fits with the idea that entanglement may be a more widespread property than naively expected [8-10].

This analysis faces the difficulty that there is no Hilbert state factorization because of the emergence of a quantum correlation between intensity and polarization not present in the classical realm. So rigorous and complete conclusions can be obtained only after embedding the system space in a larger space to make room so these variables become truly independent having their own Hilbert spaces.

\footnotetext{
*alluis@ fis.ucm.es; http://www.ucm.es/info/gioq
}

Therefore, this variablelike entanglement should be probably better described as protoentanglement that may be eventually converted into particle entanglement via the coupling of the system space with an ancilla system. This would effectively simulate the above embedding of the system space.

We think there is still much to be understood about entanglement, as revealed by classical entanglement [11] and fruitful emerging connections between classical and quantum optics [12]. This can shed a lot of light on the quantum to classical borderline with interesting consequences, both from fundamental perspectives and technological applications.

\section{SETTINGS}

Our state space is made of two modes of the electromagnetic field described by the complex amplitude operators $a_{1,2}$ acting on the corresponding component of the product Hilbert space $\mathcal{H}=\mathcal{H}_{1} \otimes \mathcal{H}_{2}$.

\section{A. Total number and relative phase operators}

The total number operator is $N=a_{1}^{\dagger} a_{1}+a_{2}^{\dagger} a_{2}$. Relative phase variables can be constructed via the Stokes operators [13]

$$
S_{x}=a_{1}^{\dagger} a_{2}+a_{2}^{\dagger} a_{1}, \quad S_{y}=i\left(a_{2}^{\dagger} a_{1}-a_{1}^{\dagger} a_{2}\right), \quad S_{z}=a_{1}^{\dagger} a_{1}-a_{2}^{\dagger} a_{2}
$$

that satisfy the commutation relations

$$
\left[N, S_{j}\right]=0, \quad\left[S_{x}, S_{y}\right]=2 i S_{z},
$$

for $j=x, y, z$, and cyclic permutations, respectively. For example a suitable unitary operator exponential of the phase difference can be constructed via a polar decomposition of the $S_{ \pm}$operators $S_{ \pm}=S_{x} \pm i S_{y}$ [14]. Alternatively, there is a nonunitary solution as product of the corresponding SusskindGlogower operators $E_{1} E_{2}^{\dagger}$ with

$$
E_{j}|n\rangle=|n-1\rangle_{j}, \quad E_{j}|0\rangle_{j}=0,
$$

where $|n\rangle_{j}$ represents the photon-number basis in $\mathcal{H}_{j}$. The Stokes operators are basic for the description of any twobeam interferometer. Naturally they describe as well quantum 
polarization properties [15]. Interference and polarization are isomorphic phenomena and we may equally refer to one or the other simply depending on whether the modes $a_{1,2}$ represent the same vibration state or orthogonal vibrations.

In principle we need not specify the concrete form of the relative-phase or polarization operator, and we may refer to it in a simple generic form as $M$, since we will only use that $[N, M]=0$. Nevertheless, for definiteness we will mainly illustrate the procedure with $M=S_{z}$.

\section{B. Hilbert-space factorization}

Naturally, entanglement properties are closely related to the Hilbert space structure and the nature of the observables chosen [16]. In our case, the presence of the operator $N$ in the pair of commuting variables suggests the following change of labels in the photon-number basis:

$$
|n, m\rangle=\left|n_{1}=\frac{n}{2}+m\right\rangle_{1}\left|n_{2}=\frac{n}{2}-m\right\rangle_{2},
$$

where

$$
n=n_{1}+n_{2}, \quad m=\frac{n_{1}-n_{2}}{2} .
$$

Note that for each $n$ the range of possible values for $m$ is bounded and ranges as $m=-n / 2,-n / 2+1, \ldots, n / 2$. This forces the following split sum of the Hilbert space:

$$
\mathcal{H}=\bigoplus_{n=0}^{\infty} \mathcal{H}_{n}
$$

where $\mathcal{H}_{n}$ is the finite-dimensional Hilbert space of dimension $n+1$ spanned by the basis vectors $|n, m\rangle$ with fixed $n$.

This means a rather unnatural link between total energy and relative phase or polarization, which is universal, independent of the field state. This is that the spectrum of any $M$ in general depends on the value of $N$. This is clearly so for the phasedifference operator with eigenvalues $2 \pi /(N+1)$ [17], and this is behind the Heisenberg limit in quantum metrology. There seems to be no fundamental reason for this purely quantum feature without classical analog. For example, in the classical domain any polarization state is allowed for any field intensity since they are clearly independent degrees of freedom.

This basic quantum link between total number and relative phase or polarization prevents any Hilbert-state factorization of the form $\mathcal{H}=\mathcal{H}_{N} \otimes \mathcal{H}_{M}$. This has consequences when assessing entanglement of the $N, M$ variables since typical entanglement measures are devised for the cases of Hilbert-state factorization, essentially via partial traces, not easily addressed otherwise. Nevertheless, there is an alternative strategy that avoids this difficulty by embedding $\mathcal{H}_{1} \otimes \mathcal{H}_{2}$ in a larger space making room to remove the link between variables. This will be examined in more detail in Sec. IV.

\section{States}

The main state we are going to consider is the product of Glauber coherent states $\left|\alpha_{1}\right\rangle_{1}\left|\alpha_{2}\right\rangle_{2}$ as eigenstates of the complex-amplitude operators $a_{j}\left|\alpha_{j}\right\rangle_{j}=\alpha_{j}\left|\alpha_{j}\right\rangle_{j}$, with, in the number basis,

$$
|\alpha\rangle=e^{-|\alpha|^{2} / 2} \sum_{n=0}^{\infty} \frac{\alpha^{n}}{\sqrt{n !}}|n\rangle .
$$

Clearly there is no entanglement between the $a_{1,2}$ variables, nor between any pair of variables derived from them under energy conserving canonical transformations. The scenario is different for total number and relative phase variables as we will see in Sec. III.

To this end it may be illustrative to express $\left|\alpha_{1}\right\rangle_{1}\left|\alpha_{2}\right\rangle_{2}$ in the $|n, m\rangle$ basis as

$$
\left|\alpha_{1}\right\rangle_{1}\left|\alpha_{2}\right\rangle_{2}=\sum_{n=0}^{\infty} \sqrt{p_{n}} e^{i n \delta}|n, \Omega\rangle,
$$

where $|n, \Omega\rangle$ are the $\mathrm{SU}(2)$ coherent states [18]

$$
\begin{aligned}
|n, \Omega\rangle= & \sum_{m=-j}^{j} \sqrt{\frac{(2 j) !}{(j-m) !(j+m) !}} \\
& \times \sin ^{j+m} \frac{\theta}{2} \cos ^{j-m} \frac{\theta}{2} e^{i 2 m \phi}|n, m\rangle,
\end{aligned}
$$

and $p_{n}$ is the probability of having a total photon number $n$,

$$
p_{n}=e^{-\bar{N}} \frac{\bar{N}^{n}}{n !},
$$

with $\bar{N}=\left|\alpha_{1}\right|^{2}+\left|\alpha_{2}\right|^{2}$ being the total mean number of photons. This expression follows after the change of variables

$$
\alpha_{1}=r \sin \frac{\theta}{2} e^{i \delta} e^{i \phi}, \quad \alpha_{2}=r \cos \frac{\theta}{2} e^{i \delta} e^{-i \phi},
$$

with $r^{2}=\bar{N}$.

On the opposite side we may also consider the two-mode squeezed vacuum

$$
|\xi\rangle=\sqrt{1-|\xi|^{2}} \sum_{n=0}^{\infty} \xi^{n}|n\rangle_{1}|n\rangle_{2},
$$

with total mean number of photons $\bar{N}=2|\xi|^{2} /\left(1-|\xi|^{2}\right)$. In this state there is clearly entanglement in the $a_{1,2}$ modes. On the other hand, in the $|n, m\rangle$ basis we get that $m=0$ for all $n$, suggesting that $M$ does not depend on $N$. Thus we might expect some kind of factorization in the total number and relative phase variables.

We have the following expression in the $|n, m\rangle$ basis:

$$
|\xi\rangle=\sqrt{1-|\xi|^{2}} \sum_{n=0}^{\infty} \xi^{n}|2 n, 0\rangle
$$

and the following probability of having a total photon number $2 n$,

$$
p_{2 n}=\frac{2 \bar{N}^{n}}{(\bar{N}+2)^{n+1}} .
$$

\section{ENTANGLEMENT BETWEEN TOTAL NUMBER AND RELATIVE PHASE}

We examine the entanglement between total number $N$ and half the number difference $M$, this is the $n, m$ variables. This is addressed from the perspective of two different entanglement 
criteria valid for pure states, linear entropy and covariance. This procedure is completely general and could be equally well applied to any pair of commuting observables $A$ and $B$ in the form $N=A+B$ and $M=A-B$. The interest of choosing number operators lies on their simplicity both for theoretical and practical reasons.

\section{A. Linear entropy}

For pure states entanglement is clearly recognized by taking the trace with respect to one of the subsystems. In the case of factorization such reduced state, say $\rho_{R}$, is pure and mixed otherwise. Purity is disclosed by the trace of its square $\rho_{R}^{2}$, so a good measure of entanglement is $S=1-\operatorname{tr}\left(\rho_{R}^{2}\right)$. For factorized states we have $S=0$ while maximal entanglement corresponds to $S=1$.

Within this scenario let us address alternatively the partial trace with respect to the total number variable $N$ or half the number difference $M$. Even if there is no Hilbert-state splitting $\mathcal{H}_{N} \otimes \mathcal{H}_{M}$, we calculate the closest analog to the idea of subsystem trace allowed in this case in order to find possible entanglement. A more rigorous approach will be provided later via an enlargement of the Hilbert space.

We consider arbitrary pure states, expressed in the photonnumber basis and in the total number and half the number difference as

$$
|\psi\rangle=\sum_{k, \ell} c_{k, \ell}|k\rangle_{1}|\ell\rangle_{2}=\sum_{n=0}^{\infty} \sum_{m=-\frac{n}{2}}^{\frac{n}{2}} c_{\frac{n}{2}+m, \frac{n}{2}-m}|n, m\rangle .
$$

We can restrict the total density matrix $\rho=|\psi\rangle\langle\psi|$ to the subalgebra generated by $N$ making use of the closest analog to the partial trace with respect to total number. This is done by removing all the coherences between different $n, n^{\prime}$ values, so only the minimal information required to compute the statistics of any operator $M$ commuting with $N$ is left. This is

$$
\rho_{M}=\sum_{n=0}^{\infty} \Pi_{n} \rho \Pi_{n}=\sum_{n=0}^{\infty}\left|\psi_{n}\right\rangle\left\langle\psi_{n}\right|
$$

where $\Pi_{n}$ are orthogonal projectors on the subspaces of definite total photon number $\mathcal{H}_{n}$,

$$
\Pi_{n}=\sum_{m=-\frac{n}{2}}^{\frac{n}{2}}|n, m\rangle\langle n, m|
$$

and $\left|\psi_{n}\right\rangle=\Pi_{n}|\psi\rangle$ are the corresponding unnormalized projections with

$$
\left\langle\psi_{n} \mid \psi_{n^{\prime}}\right\rangle=p_{n} \delta_{n, n^{\prime}}, \quad p_{n}=\sum_{m=-\frac{n}{2}}^{\frac{n}{2}}\left|c_{\frac{n}{2}+m, \frac{n}{2}-m}\right|^{2},
$$

and $p_{n}$ is the probability of having a total photon number $n$.

Similarly, the closest analog of the partial trace with respect to the phaselike or polarization variables is

$$
\rho_{N}=\sum_{m=-\infty}^{\infty} \Pi_{m} \rho \Pi_{m}=\sum_{m=-\infty}^{\infty}\left|\psi_{m}\right\rangle\left\langle\psi_{m}\right|,
$$

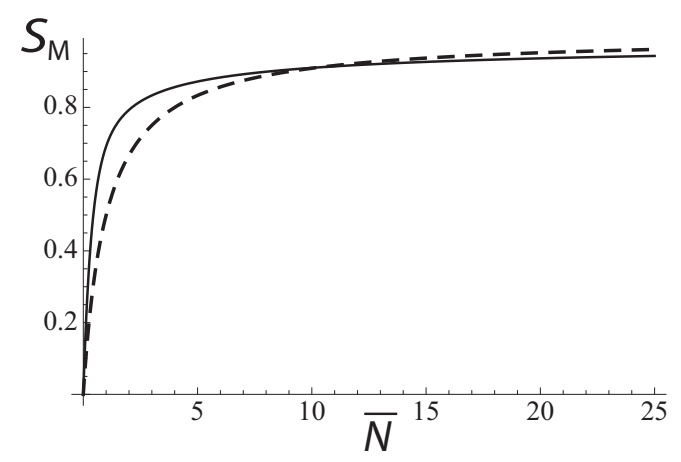

FIG. 1. Linear entropy $S_{M}$ versus mean number of photos $\bar{N}$ for a two-mode coherent state (solid line) and a two-mode squeezed vacuum state (dashed line).

where $\Pi_{m}$ are orthogonal projectors on the subspaces of definite value $m$ of $M=S_{z} / 2$,

$$
\Pi_{m}=\sum_{n=2|m|}^{\infty}|n, m\rangle\langle n, m|,
$$

and $\left|\psi_{m}\right\rangle=\Pi_{m}|\psi\rangle$ are the corresponding unnormalized projections with

$$
\left\langle\psi_{m} \mid \psi_{m^{\prime}}\right\rangle=p_{m} \delta_{m, m^{\prime}}, \quad p_{m}=\sum_{n=2|m|}^{\infty}\left|c_{\frac{n}{2}+m, \frac{n}{2}-m}\right|^{2},
$$

and $p_{n}$ is the probability of having a total value of $M$ equal to $m$.

With this we can compute the corresponding linear entropies for the $\rho_{M}$ and $\rho_{N}$, leading to

$$
S_{M}=1-\sum_{n=0}^{\infty} p_{n}^{2}, \quad S_{N}=1-\sum_{m=-\infty}^{\infty} p_{m}^{2} .
$$

Note that in general $S_{N} \neq S_{M}$ once again due to the lack of the corresponding Hilbert-space splitting.

In the case of the two-mode coherent state $\left|\alpha_{1}\right\rangle_{1}\left|\alpha_{2}\right\rangle_{2}$ we have $p_{n}$ given by Eq. (2.10) and then

$$
S_{M}=1-e^{-2 \bar{N}} I_{0}(2 \bar{N})
$$

where $I_{0}$ is the Bessel function of order zero. The entropy is plotted against $\bar{N}$ in Fig. 1, showing the entanglement increases as $\bar{N}$ increases with $S_{M} \rightarrow 1$ when $\bar{N} \rightarrow \infty$. On the other hand for the range of values examined we have seen numerically that $S_{N} \simeq S_{M}$ to the extreme of being indistinguishable.

On the other hand, for the two-mode squeezed vacuum (2.12) we have $p_{n}$ given by Eq. (2.14) leading to

$$
S_{M}=\frac{\bar{N}}{1+\bar{N}},
$$

with the same behavior than coherent states as shown in Fig. 1, maybe surprisingly. On the other hand, the result is the opposite for $S_{N}$, since we clearly have that $\rho_{N}$ is actually a pure state $\left|\psi_{m=0}\right\rangle$ so that $S_{N}=0$.

In principle it might be questioned whether these mixedness properly reflect entanglement, after the lack of Hilbert-space factorization. We think that at least they may provide a clear indicator. Maybe more rigorous and conclusive results will be 
provided in Sec. IV when embedding the two-mode space in a larger Hilbert space so there is room for the Hilbert-space factorization.

\section{B. Covariance}

Let us now use the covariance criterion studied in Ref. [8] that establishes that for pure states there is entanglement between $A$ and $B$ variables provided that

$$
\langle A B\rangle \neq\langle A\rangle\langle B\rangle \text {. }
$$

Clearly this correlation is incompatible with state factorization if $A$ and $B$ act on different spaces. Let us extend this idea to the pair of commuting variables we are considering in this work. Let this time be

$$
A=N=a_{1}^{\dagger} a_{1}+a_{2}^{\dagger} a_{2}, \quad B=S_{z}=a_{1}^{\dagger} a_{1}-a_{2}^{\dagger} a_{2} .
$$

So

$$
\langle A B\rangle-\langle A\rangle\langle B\rangle=\Delta^{2} a_{1}^{\dagger} a_{1}-\Delta^{2} a_{2}^{\dagger} a_{2},
$$

so that this criterion predicts entanglement provided that $\Delta^{2} a_{1}^{\dagger} a_{1} \neq \Delta^{2} a_{2}^{\dagger} a_{2}$.

For the pair coherent states $\Delta^{2} a_{j}^{\dagger} a_{j}=\left|\alpha_{j}\right|^{2}$, so that there is entanglement provided that $\left|\alpha_{1}\right| \neq\left|\alpha_{2}\right|$. Otherwise we get entanglement by replacing $S_{z}$ by another Stokes operator.

On the other hand, for the two-mode squeezed vacuum state (2.12) the equality is always satisfied for all $S_{j}$,

$$
\left\langle N S_{j}\right\rangle=0,\left\langle S_{j}\right\rangle=0 .
$$

Nevertheless it fails to be satisfied for higher power operators, such as

$$
\left\langle N S_{x}^{2}\right\rangle=\bar{N}(\bar{N}+2)(3 \bar{N}+2)
$$

while

$$
\left\langle S_{x}^{2}\right\rangle=\bar{N}(\bar{N}+2) .
$$

Finally we may consider the rather odd case $A=B$, where we have entanglement of $A$ with itself provided that $\Delta^{2} A \neq 0$. We explain this striking case in Sec. V.

\section{HILBERT-SPACE FACTORIZATION VIA EMBEDDING}

In principle, the above claims about entanglement may be obscured by the lack of Hilbert-space factorization of the form $\mathcal{H}=\mathcal{H}_{N} \otimes \mathcal{H}_{M}$. This rather technical point can be avoided by extending the state space so that $\mathcal{H}_{1} \otimes \mathcal{H}_{2} \subset \mathcal{H}_{N} \otimes \mathcal{H}_{M}$ and considering that meaningful states are restricted to some physical sector of $\mathcal{H}_{N} \otimes \mathcal{H}_{M}$. Although these observables are nonlocal, they can be measured and tailored theoretically as shown in [19].

Thus we consider the following embedding of $\mathcal{H}_{1} \otimes \mathcal{H}_{2}$ into the product of two Hilbert spaces $\mathcal{H}_{N} \otimes \mathcal{H}_{M}$ via the following injective correspondence between basis vectors:

$$
\left|n_{1}\right\rangle_{1}\left|n_{2}\right\rangle_{2} \rightarrow|n\rangle_{N}|m\rangle_{M}
$$

with

$$
n=n_{1}+n_{2}, \quad m=\frac{n_{1}-n_{2}}{2} .
$$

This is essentially the same basis relabeling in Eqs. (2.4) and (2.5), but now we admit that there is no restriction on the $n, m$ values in $|n\rangle_{N}|m\rangle_{M}: n$ runs over all integers while $m$ runs over all integers and half-integers. In other words, the correspondence (4.1) does not exhaust all basis vectors $|n\rangle_{N}|m\rangle_{M}$ and there are states $|n\rangle_{N}|m\rangle_{M}$ without preimage. The physical sector holds for basis vector with integer and nonnegative $\frac{n}{2} \pm m$.

This is essentially a version of previous expansions of the state space to formally include negative numbers in the number basis. This has been used in the quantum phase context to recover the unitarity of the Susskind-Glogower operators, so that $E|0\rangle=|-1\rangle$ and so on [20]. Then it is said that physical states are restricted to the sector of nonnegative integers. So it is natural to find that this strategy is also useful in the context dealing also with number-phase variables.

Now the operation of taking the partial trace with respect to the $N$ variable for a pure state of the form (3.1) is quite transparent leading to

$$
\rho_{M}=\sum_{m, m^{\prime}=-\infty}^{\infty} d_{m, m^{\prime}}|m\rangle_{M}\left\langle m^{\prime}\right|,
$$

where

$$
d_{m, m^{\prime}}=\sum_{n=-\infty}^{\infty} c_{\frac{n}{2}+m, \frac{n}{2}-m} c_{\frac{n}{2}+m^{\prime}, \frac{n}{2}-m^{\prime}}^{*}
$$

The corresponding trace of the square of $\rho_{M}$ gives the following linear entropy:

$$
S=1-\sum_{m, m^{\prime}=-\infty}^{\infty}\left|d_{m, m^{\prime}}\right|^{2}
$$

Note that in general this entropy is slightly different than the ones in Sec. III A since we are now in a different space. We notice also that now there can be no distinction between the $N$ and $M$ traces.

Let us compute $S$ for the pair coherent state $\left|\alpha_{1}\right\rangle_{1}\left|\alpha_{2}\right\rangle_{2}$ with $\alpha_{2}=0$, this is the vacuum in mode $a_{2}$. In such a case $c_{k, \ell}=$ 0 unless $\ell=0$ that leads to $m=m^{\prime}=n / 2$ in Eq. (4.4) and $d_{m, m^{\prime}}=d_{n / 2, n / 2}=p_{n}$ with the same $p_{n}$ in Eq. (2.10), so that

$$
\rho_{M}=\sum_{n=0}^{\infty} e^{-\bar{N}} \frac{\bar{N}^{n}}{n !}|n / 2\rangle_{M}\langle n / 2| .
$$

Therefore we have the same result in Eq. (3.9). However, in this extended case the entropy $S$ no longer depends just on $\bar{N}$ and the result is different under different splittings of the photons between modes. This is for example the case $\alpha_{1}=\alpha_{2}=1$ for which we have $S=0.60$. This is below the value obtained for the same state in Sec. III. Besides, the entanglement for $\mathcal{H}_{1} \otimes \mathcal{H}_{2}$ is always greater than in $\mathcal{H}_{N} \otimes \mathcal{H}_{M}$. This is due to the number of terms in the coefficients in $\mathcal{H}_{1} \otimes \mathcal{H}_{2}$ is less than in $\mathcal{H}_{N} \otimes \mathcal{H}_{M}$ because of the embedding. In the physical sector $\mathcal{H}_{N} \otimes \mathcal{H}_{M}$ there are no terms of the form $|n\rangle_{N}|m\rangle_{M}$ with $|m|>n$. So we have that $S_{\mathcal{H}_{1} \otimes \mathcal{H}_{2}} \geqslant S_{\mathcal{H}_{N} \otimes \mathcal{H}_{M}}$ which means that the restriction to the physical sector reduces the entanglement.

On the other hand, for the two-mode squeezed vacuum (2.12) we get

$$
|\psi\rangle \rightarrow|\varphi\rangle_{N}|0\rangle_{M}
$$


where $|\varphi\rangle_{N}$ is an eigenstate of the square of the SusskindGlogower phase operator in the space $\mathcal{H}_{N}$,

$$
|\varphi\rangle_{N}=\sqrt{1-|\xi|^{2}} \sum_{n=0}^{\infty} \xi^{n}|2 n\rangle_{M} .
$$

In this case there is no entanglement and $S=0$ as it could be expected.

\section{FROM PROTOENTANGLEMENT TO ENTANGLEMENT}

In this section we present the conversion of protoentanglement into actual particle entanglement. This is accomplished by the coupling of the system with an appropriate ancilla system. This is a kind of operational simulation of the systemspace embedding discussed in the preceding section.

\section{A. Two-level atom entanglement}

We can provide a very specific and operational scheme considering the nonresonant interaction of the field modes with a pair of two-level atoms. Let us couple an atom to the variable $N$ and the other one to the variable $M=S_{z}$ with the following interaction Hamiltonian:

$$
H_{\mathrm{int}}=\hbar \lambda\left(N \sigma_{1}+S_{z} \sigma_{2}\right),
$$

or equivalently

$$
H_{\text {int }}=\hbar \lambda\left[a_{1}^{\dagger} a_{1}\left(\sigma_{1}+\sigma_{2}\right)+a_{2}^{\dagger} a_{2}\left(\sigma_{1}-\sigma_{2}\right)\right],
$$

where $\lambda$ is a coupling constant and $\sigma_{j}=|e\rangle_{j}\langle e|$, with $|g, e\rangle_{j}$ being the corresponding ground and excited states, respectively. This is a really experimentally feasible field-atom coupling as already demonstrated in beautiful experiments via Rydberg interferometry [21].

The atoms are initially prepared in the state $|+\rangle_{1}|-\rangle_{2}$ with

$$
| \pm\rangle_{j}=\frac{1}{\sqrt{2}}\left(|g\rangle_{j} \pm|e\rangle_{j}\right)
$$

The key point is that when $N$ is even (odd) $S_{z}$ is also even (odd). We fix the interaction time $\tau$ to be such that $\lambda \tau=\pi$ so that when $N$ and $S_{z}$ are even the evolution takes the initial state again to the initial state

$$
|+\rangle_{1}|-\rangle_{2} \longrightarrow|+\rangle_{1}|-\rangle_{2},
$$

while when $N$ and $S_{z}$ are odd the evolution produces the following transformation on the atomic state:

$$
|+\rangle_{1}|-\rangle_{2} \longrightarrow|-\rangle_{1}|+\rangle_{2} \text {. }
$$

Therefore, if the initial state is $|\psi\rangle|+\rangle_{1}|-\rangle_{2}$ the evolved state at time $\tau$ is

$$
\left|\psi_{e}\right\rangle|+\rangle_{1}|-\rangle_{2}+\left|\psi_{o}\right\rangle|-\rangle_{1}|+\rangle_{2},
$$

where $\left|\psi_{e, o}\right\rangle$ are the unnormalized orthogonal projections of the initial field state $|\psi\rangle$ on the subspaces of even and odd total photon number $N$, respectively, with

$$
|\psi\rangle=\left|\psi_{e}\right\rangle+\left|\psi_{o}\right\rangle, \quad\left\langle\psi_{e} \mid \psi_{o}\right\rangle=0
$$

Note that the state in Eq. (5.6) is a tripartite entangled state. In order to extract pure particle entanglement in the atomic space we may project the field system on a suitable state. For

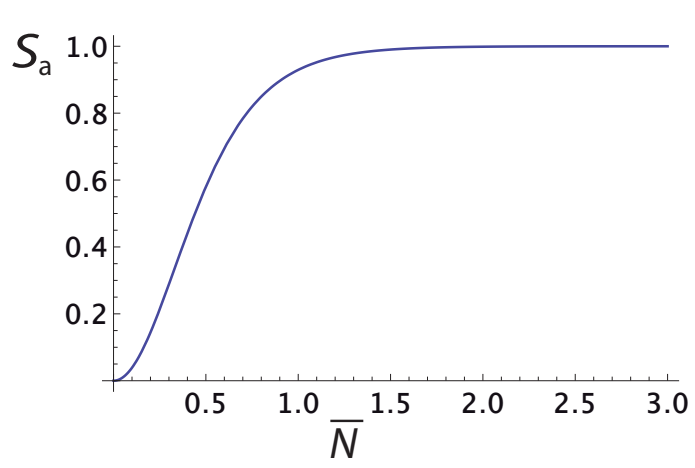

FIG. 2. The atomic linear entropy $S_{a}$ versus mean number of photos $\bar{N}$ for a two-mode coherent state.

simplicity this may be the same initial state $|\psi\rangle$, so that the reduced atomic state reads

$$
\frac{1}{\sqrt{p_{e}^{2}+p_{o}^{2}}}\left(p_{e}|+\rangle_{1}|-\rangle_{2}+p_{o}|-\rangle_{1}|+\rangle_{2}\right)
$$

where

$$
p_{e, o}=\left\langle\psi_{e, o} \mid \psi_{e, o}\right\rangle .
$$

We can assess the entanglement of this reduced state via a properly normalized version of the linear entropy as $S_{a}=$ $2\left[1-\operatorname{tr}\left(\rho_{R}^{2}\right)\right]$ so that it ranges between 0 and 1 with

$$
S_{a}=2\left[1-\frac{p_{e}^{4}+p_{o}^{4}}{\left(p_{e}^{2}+p_{o}^{2}\right)^{2}}\right] .
$$

We have $S_{a}=0$ for factorized states $p_{e}=0$ or $p_{o}=0$ while $S_{a}=1$ for maximally entangled states $p_{e}=p_{o}=1 / 2$.

In the case of product of coherent states it holds that $p_{e, o}$ depend only on the total mean number of photons $\bar{N}$, as shown by Eqs. (2.8) and (2.10) [18]. More specifically

$$
p_{e}=e^{-\bar{N}} \sinh \bar{N}, \quad p_{o}=e^{-\bar{N}} \cosh \bar{N},
$$

so that

$$
S_{a}=\tanh ^{2}(2 \bar{N})
$$

Moreover, we have that the condition for maximally entangled states is reached actually for very small mean numbers and $p_{e} \simeq p_{o} \simeq 1 / 2$ for $\bar{N}$ as small as $\bar{N}=2$ leading to $S_{a}=0.998$. In Fig. 2 we have plotted $S_{a}$ as a function of $\bar{N}$. Within this same scenario, when the initial field state is the two-mode vacuum (2.12) we have only an even number of photons $p_{o}=0$, the atomic state always factorizes, and $S_{a}=0$. So there is a very large agreement between this operational entanglement conversion and the above analysis.

\section{B. Covariance criterion}

Let us show that the protoentanglement revealed by criterion (3.11) has a complete equivalence with particle entanglement as far as one attempts to actually measure both $A$ and $B$ simultaneously to compute $\langle A B\rangle$. To show this we consider that the system space is enlarged from $\mathcal{H}$ to $\mathcal{H} \otimes \mathcal{H}_{a}$ in order to make room for a joint measurement of $A$ and $B$, always with $[A, B]=0$. For example we consider that $A$ is measured in the original system $\mathcal{H}$ while $B$ is measured in an different system 
$\mathcal{H}_{a}$ prepared in some ancilla state $|\varphi\rangle_{a}$. In order to transfer information about $B$ from $\mathcal{H}$ to $\mathcal{H}_{a}$ we consider the following state transformation:

$$
|\psi\rangle|\varphi\rangle_{a} \rightarrow|\Psi\rangle=e^{i B Q_{a}}|\psi\rangle|\varphi\rangle_{a},
$$

where $Q_{a}$ is some operator acting solely on $\mathcal{H}_{a}$. Let the observable to be measured in $\mathcal{H}_{a}$ be $P_{a}$ with

$$
e^{-i b Q_{a}} P_{a} e^{i b Q_{a}}=P_{a}+b, \quad{ }_{a}\left\langle\varphi\left|P_{a}\right| \varphi\right\rangle_{a}=0,
$$

for every real scalar $b$.

With all this we get

$$
\left\langle\Psi\left|A P_{a}\right| \Psi\right\rangle=\langle\psi|A B| \psi\rangle
$$

and

$$
\langle\Psi|A| \Psi\rangle=\langle\psi|A| \psi\rangle, \quad\left\langle\Psi\left|P_{a}\right| \Psi\right\rangle=\langle\psi|B| \psi\rangle .
$$

Thus, the variablelike entanglement criterion in $|\psi\rangle$ for $A, B$ is fully equivalent to the particlelike entanglement criterion in the state $|\Psi\rangle$ for $A$ and $P_{a}$.

This particle entanglement may naturally arise even if we measure the same observable twice if $\Delta^{2} A \neq 0$. Now we see that there is nothing striking in the entanglement of the state $e^{i A Q_{a}}|\psi\rangle|\varphi\rangle_{a}$.

\section{CONCLUSIONS}

We have investigated the entanglement between total number and polarization or relative-phase variables arising in two-mode electromagnetic fields. Since these variables lack a definite splitting of Hilbert spaces this entanglement should be better considered as a kind of protoentanglement. Nevertheless we have shown that this protoentanglement can be actually extracted and converted into real particle entanglement via feasible experimental procedures. So this might be regarded as a useful and practical entanglement resource. This is because we have shown that this holds also for products of Glauber coherent states, confirming previous results indicating nonclassical features of coherent states in phase-number variables.

\section{ACKNOWLEDGMENTS}

A.L. acknowledges financial support from Spanish Ministerio de Economía y Competitividad Project No. FIS201675199-P, and from the Comunidad Autónoma de Madrid research consortium QUITEMAD+ Grant No. S2013/ICE2801.
[1] A. Aiello, F. Töppel, Ch. Marquardt, E. Giacobino, and G. Leuchs, Quantum-like nonseparable structures in optical beams, New J. Phys. 17, 043024 (2015).

[2] J. Sahota and N. Quesada, Quantum correlations in optical metrology: Heisenberg-limited phase estimation without mode entanglement, Phys. Rev. A 91, 013808 (2015).

[3] A. Fine, Hidden Variables, Joint Probability, and the Bell Inequalities, Phys. Rev. Lett. 48, 291 (1982); A. Rivas, Incompatible statistics and Bell-KS theorem, arXiv:1501.04929.

[4] J. Vaccaro, Number-phase Wigner function on Fock space, Phys. Rev. A 52, 3474 (1995); A. Luis, Nonclassical polarization states, ibid. 73, 063806 (2006).

[5] A. Luis, Nonclassical light revealed by the joint statistics of simultaneous measurements, Opt. Lett. 41, 1789 (2016).

[6] A. Luis and L. Monroy, Nonclassicality of coherent states: Entanglement of joint statistics, Phys. Rev. A 96, 063802 (2017).

[7] L. M. Johansen, Nonclassical properties of coherent states, Phys. Lett. A 329, 184 (2004); Nonclassicality of thermal radiation, J. Opt. B 6, L21 (2004); L. M. Johansen and A. Luis, Nonclassicality in weak measurements, Phys. Rev. A 70, 052115 (2004).

[8] A. C. de la Torre, D. Goyeneche and L. Leitao, Entanglement for all quantum states, Eur. J. Phys. 31, 325 (2010).

[9] M. Kupczynski, On operational approach to entanglement and how to certify it, arXiv:1602.00146.

[10] A. Ferraro, L. Aolita, D. Cavalcanti, F. M. Cucchietti, and A. Acín, Almost all quantum states have nonclassical correlations, Phys. Rev. A 81, 052318 (2010).

[11] R. J. C. Spreeuw, A classical analogy of entanglement, Found. Phys. 28, 361 (1998); A. Luis, Coherence, polarization, and entanglement for classical light fields, Opt. Commun. 282, 3665 (2009); X.-F. Qian and J. H. Eberly, Entanglement and classical polarization states, Opt. Lett. 36, 4110 (2011); K. H. Kagalwala, G. Di Giuseppe, A. F. Abouraddy, and B. E. A. Saleh, Bell measure in classical optical coherence, Nat. Photon. 7, 72 (2013); F. De Zela, Relationship between the degree of polarization, indistinguishability, and entanglement, Phys. Rev. A 89, 013845 (2014); F. Töppel, A. Aiello, Ch. Marquardt, E. Giacobino, and G. Leuchs, Classical entanglement in polarization metrology, New J. Phys. 16, 073019 (2014).

[12] J. H. Eberly, X.-F. Qian, A. Al Qasimi, H. Ali, M. A. Alonso, R. Gutiérrez-Cuevas, B. J. Little, J. C. Howell, T. Malhotra, and A. N. Vamivakas, Quantum and classical optic-emerging links, Phys. Scr. 91, 063003 (2016); X.-F. Qian, A. N. Vamivakas, and J. H. Eberly, Emerging Connections: Classical and Quantum Optics, Opt. Photon. News (2017).

[13] J. Schwinger, Quantum Theory of Angular Momentum (Academic, New York, 1965).

[14] A. Luis and L. L. Sanchez-Soto, Phase-difference operator, Phys. Rev. A 48, 4702 (1993); Quantum phase difference, phase measurements and Stokes operators, Prog. Opt. 41, 421 (2000).

[15] A. Luis, Polarization in quantum optics, Prog. Opt. 61, 283 (2016).

[16] P. Zanardi, D. A. Lidar, and S. Lloyd, Quantum Tensor Product Structures are Observable Induced, Phys. Rev. Lett. 92, 060402 (2004); N. L. Harshman and S. Wickramasekara, Tensor product structures, entanglement, and particle scattering, Open Syst. Inf. Dyn. 14, 341 (2007).

[17] A. Luis and J. Peřina, Optimum phase-shift estimation and the quantum description of the phase difference, Phys. Rev. A 54, 4564 (1996). 
[18] P. W. Atkins and J. C. Dobson, Angular momentum coherent states, Proc. R. Soc. London Ser. A 321, 321 (1971); F. T. Arecchi, E. Courtens, R. Gilmore, and H. Thomas, Atomic coherent states in quantum optics, Phys. Rev. A 6, 2211 (1972); O. Giraud, P. Braun, and D. Braun, Classicality of spin states, ibid. 78, 042112 (2008); Quantifying quantumness and the quest for queens of quantum, New J. Phys. 12, 063005 (2010).

[19] Z. Q. Wu, H. Cao, H. L. Zhang, S. J. Ma, and J. H. Huang, Experimental proposal for performing nonlocal measurement of a product observable, Opt. Express 24, 27331 (2016).

[20] R. G. Newton, Quantum action-angle variables for harmonic oscillators, Ann. Phys. (NY) 124, 327 (1980); S. M. Barnett and D. T. Pegg, Phase in quantum optics, J. Phys. A 19, 3849 (1986).

[21] M. Brune, S. Haroche, V. Lefevre, J. M. Raimond, and N. Zagury, Quantum Nondemolition Measurement of Small Photon Numbers by Rydberg-Atom Phase-Sensitive Detection, Phys. Rev. Lett. 65, 976 (1990); M. Brune, S. Haroche, J. M. Raimond,
L. Davidovich, and N. Zagury, Manipulation of photons in a cavity by dispersive atom-field coupling: Quantum-nondemolition measurements and generation of Schrödinger cat states, Phys. Rev. A 45, 5193 (1992); M. Brune, E. Hagley, J. Dreyer, X. Maître, A. Maali, C. Wunderlich, J. M. Raimond, and S. Haroche, Observing the Progressive Decoherence of the Meter in a Quantum Measurement, Phys. Rev. Lett. 77, 4887 (1996); S. Haroche, Entanglement, decoherence and the quantum/classical boundary, Phys. Today 51(7), 36 (1998); J. M. Raimond, M. Brune, and S. Haroche, Reversible Decoherence of a Mesoscopic Superposition of Field States, Phys. Rev. Lett. 79, 1964 (1997); L. Davidovich, A. Maali, M. Brune, J. M. Raimond, and S. Haroche, Quantum Switches and Nonlocal Microwave Fields, ibid. 71, 2360 (1993); L. Davidovich, M. Brune, J. M. Raimond, and S. Haroche, Mesoscopic quantum coherences in cavity QED: Preparation and decoherence monitoring schemes, Phys. Rev. A 53, 1295 (1996). 